\title{
Combined Use of Acid Fibroblast Growth Factor, Granulocyte Colony-stimulating Factor and Zinc Sulphate Accelerates Diabetic Ulcer Healing
}

\author{
Shaoqiang Lin, ${ }^{a, b},{ }^{\prime}$ Hengwu Xu, ${ }^{a, b}, \#$ Jian Xiao,,${ }^{a, b}$ Yanlong Liu, ${ }^{a, b}$ Yi Zhang, ${ }^{a}$ \\ Lu Cai, ${ }^{a, c}$ Xiaokun Li, ${ }^{*, a, b}$ and Yi Tan ${ }^{a, b, c}$ \\ ${ }^{a}$ The Chinese-American Research Institute for Diabetic Complications, ${ }^{b}$ Key Laboratory of Biotechnology Pharmaceutical Engineering \\ Wenzhou Medical College, Chashan College Park, Wenzhou 325035, China and ${ }^{c}$ Department of Pediatrics, the University of Louisville, \\ 570 South Preston Street, Suite 311, Louisville, KY 40202, U.S.A.
}

(Received June 29, 2009; Accepted August 17, 2009)

\begin{abstract}
Our previous studies demonstrated that topic application of recombinant human acid fibroblast growth factor (aFGF) significantly, but still not completely, improved in diabetic ulcer healing. To obtain a maximal therapy for diabetic ulcer healing, a combined protocol containing aFGF, anti-oxidative reagent zinc (Zn) and stem cell stimulator granulocyte colony-stimulating factor (G-CSF), i.e.: aFGF/G-CSF/zinc sulphate $\left(\mathrm{ZnSO}_{4}\right)$, was explored in the present study. Diabetes was induced by a single dose of streptozotocin (STZ, $55 \mathrm{mg} / \mathrm{kg}$ ) in Sprague Dawley rats, and full thickness skin wound was made in diabetic rats at 2 months after diabetes onset. Diabetic ulcer rats were treated with aFGF, G-CSF, $\mathrm{ZnSO}_{4}$, aFGF/G-CSF/ZnSO $\mathrm{Z}_{4}$ or vehicle control, respectively and 3, 6, 9, 12, 15, 18, 21 and 28 days later, therapeutic effects were evaluated by calculating ulcer area. On day 7, 14, 21 after treatment, rats were sacrificed to collect 2 pieces of dorsal skin from 3 different sites (wound center, edge and healed area) to perform histopathological examination. Results showed that treatment with aFGF/G-CSF/ZnSO 4 significantly enhanced ulcer healing compared with single drug treatment groups at different time points. Healing times for $100 \%$ of the wound in the $\mathrm{aFGF} / \mathrm{G}-\mathrm{CSF} / \mathrm{ZnSO}_{4}$ group was $20.00 \pm 1.15$ days, and significantly shorter than those in single treatment groups $(p<0.05)$. Histopathological and immunohistochemical analysis disclosed significant increases in capillary density, proliferating cells, the expression of tissue inhibitor of metalloproteinase 1 (TIMP-1) and the ratios of TIMP-1 to matrix metalloproteinase 1 (MMP-1) in aFGF/G-CSF/ZnSO 4 group as compared to other single treatments. Collectively, the combinative protocol of aFGF/G-CSF/ZnSO 4 significantly enhanced the therapeutic effect on diabetic ulcer wound, probably through the promotion of fibroblast proliferation and differentiation, enhancement of blood vessel regeneration, and up-regulation of TIMP-1 and down-regulation of MMP-1 expression in diabetic ulcer healing process.
\end{abstract}

Key words _ acid fibroblast growth factor, granulocyte colony-stimulating factor, zinc, diabetic ulcer

\section{INTRODUCTION}

Diabetic complications have become a serious issue for public health. One of these complications is the impaired-wound healing for diabetic patients. ${ }^{1,2)}$ Lack of cellular and molecular signals required for normal wound healing process such as angiogenesis, granulation tissue formation, epithelialization, and remodeling may be the reason for

\footnotetext{
*To whom correspondence should be addressed: ChineseAmerican Research Institute for Diabetic Complications, Wenzhou Medical College, Chashan College Park, Wenzhou City, Zhejiang 325035, China. Tel.: 86-13676701796; Fax: 86-57786699983; E-mail: xiaokunli@163.net

${ }^{\#}$ These authors contributed equally to the work.
}

the poor healing of diabetic wound. . 3-5) $^{13}$

Although multiple factors including hyperglycemia, hyperlipidemia and inflammation all contribute the pathogenic effect to various vascular complications in diabetes, ${ }^{6)}$ hyperglycemia plays a critical role in the widespread cellular damage since endothelial cells poorly regulate intracellular glucose and particular vulnerable to hyperglycemiaderived oxidative damage. ${ }^{7)}$ Furthermore, these pathogens also inhibit angiogenic pathways, leading to inadequate blood vessel growth and consequently delaying diabetic wound healing process.

Cytokines, especially various growth factors, provide the cellular and molecular signals necessary for normal healing process, but are deficient in di- 
abetic wounds. $^{1,3-5)}$ Topical application of several growth factors to stimulate fibroblast and endothelial cell proliferation to heal the impaired wound may enhance successful rate of wound healing. ${ }^{8-10)}$ Under appropriate pathophysiological conditions, growth factors, such as vascular endothelial growth factor (VEGF) and fibroblast growth factor (FGF), are released to initiate angiogenesis. ${ }^{11-13)}$ Impaired wound healing is a common condition in diabetes associated with a delay in progression beyond the inflammatory and proliferative phases of normal wound healing. ${ }^{11,12)}$ For example, FGF is secreted by fibroblasts, macrophages and in particular endothelial cells in response to tissue injury and plays an important role in angiogenesis. Hyperglycemiaderived glycation significantly reduces FGF's binding capacity to the tyrosine kinase receptor and activates signal transduction pathways responsible for both mitogenesis and capillary formation. ${ }^{12)}$ Therefore, significant acceleration of wound healing by topical application of various exogenous growth factors including FGF has been observed in diabetic animal models ${ }^{14-17)}$ and a few of diabetic patients. ${ }^{18)}$ However, only application of a single growth factor remains unable to obtain the maximal therapeutic purpose. ${ }^{19)}$ These studies suggest multiple mechanisms contributing to diabetes-impaired wound healing.

Indeed other alterations in wound local tissue except for the defect of growth factors also significantly affect the healing. ${ }^{13)}$ Zinc (Zn) is an essentially trace element for cell growth, proliferation, and cellular repair and signaling. ${ }^{13,20)}$ Diabetes significantly impairs $\mathrm{Zn}$ homeostasis, leading to systemic $\mathrm{Zn}$ deficiency, ${ }^{20)}$ which is proposed to associate with the impaired wound healing. ${ }^{21,22)}$ Significant benefit of topical application of $\mathrm{Zn}$ with or without other components on diabetic or nondiabetic wound healing was observed. ${ }^{13,23-26)}$ The beneficial effects of topical application of $\mathrm{Zn}$ include not only the correction of wound tissue's $\mathrm{Zn}$ deficiency, but also the enhancement of angiogenesis, decrease of inflammatory response and bacterial growth, and increase of antioxidants against diabetes-caused dermal and vessel further damage. More important, $\mathrm{Zn}$ is also able to amplify FGF functions. ${ }^{27,28)}$

Systemic abnormalities also play important roles in the delay of diabetic wound healing. ${ }^{12)} \mathrm{Re}-$ cent study shows that dysfunction of endothelial progenitor cells (EPCs) is a critical cause for the im- pairment of diabetic wound heal. ${ }^{26)}$ Administration of circulating CD34+ cells, which can function as EPCs, accelerates the revascularization and healing in the skin wound of streptozotocin (STZ)-induced diabetic mice. ${ }^{29,30)}$ An early study also showed that administration of stem-cell stimulating factor improved diabetic wound healing. ${ }^{31)}$ An recent study with topical use of VEGF also showed that the significantly improved diabetic wound healing was also mediated by systemic mobilization of bone marrow hematopoietic progenitor cells (BM-HPCs) including a population that contributes to blood vessel formation, as one of the multiple possible mechanisms. ${ }^{15)}$

Granulocyte colony-stimulating factor (G-CSF) is a colony-stimulating factor, which is produced by a number of different tissues to stimulate the bone marrow to produce granulocytes and stem cells and then mobilize these cells into the blood and wound tissue for participating the new blood vessel generation. ${ }^{32,33)}$ Therefore, in vivo application of G-CSF stimulated wound healing under non-diabetic condition. ${ }^{32,33)}$

The beneficial effects of these different approaches, such as topical application of FGF or Zn and/or systemic application of G-CSF imply that there may be a significantly synergistic stimulation of the wound healing when these multiple approaches are applied together. A few studies have preliminarily explored the combined therapeutic effects with two growth factors. ${ }^{34-37)}$ However, these studies just focused on the improvement of wound tissue growth factor defect without much consideration of wound tissue antioxidant and micronutrient improvement and systemic up-regulation of stem cell mobilization. Therefore, the purpose of the present study was to assess the curative effect on the healing-impaired ulcer in STZ-induced diabetic rats with a combined protocol, including topical application of recombinant human acid fibroblast growth factor (aFGF) and nutrient $(\mathrm{Zn})$ to correct wound local defect of both tissue growth factors and $\mathrm{Zn}$ homeostasis, and systemic application of G-CSF to peripherally mobilize the endogenous BM-HPCs. Although this hypothesis may be easily imaged to produce a more efficient therapeutic effect, its feasibility remains very interesting and urgent to be tested pre-clinically in animal model. We demonstrated that diabetic ulcer healing was found to be significantly improved by aFGF/G-CSF/zinc sulphate $\left(\mathrm{ZnSO}_{4}\right)$, as compared to other treatment 
combinations, suggesting the potential of aFGF/G$\mathrm{CSF} / \mathrm{ZnSO}_{4}$ as a new diabetic ulcer treatment strategy for clinical application.

\section{MATERIALS AND METHODS}

Diabetic Ulcer Rat Model-Male Sprague Dawley (S.D.) rats (body weight 200-230 g) were provided by Wenzhou Medical College Experimental Animal Center and kept in specific pathogen free facility. All animal procedures were approved by the University Animal Care and Use Committee, which is certified by the Chinese Association of Accreditation of Laboratory Animal Care.

To induce diabetic animal model, rats were given a single intraperitoneal (ip) injection of 55 $\mathrm{mg} / \mathrm{kg}$ STZ (Sigma-Aldrich, Shanghai, China) dissolved in sodium citrate buffer after $12 \mathrm{hr}$ fasting, and control group was injected with equal volume of $0.1 \mathrm{~mol} / \mathrm{l}$ citrate buffer $(\mathrm{pH}=4.2){ }^{38)}$ Food was supplied in $10 \mathrm{~min}$. after STZ injection. On day 7 after STZ injection, whole-blood glucose concentration was detected using a SureStep complete blood glucose monitor (LifeScan Inc., Shanghai, China). The rats with blood glucose concentration higher than $16.67 \mathrm{mmol} / \mathrm{l}(300 \mathrm{mg} / \mathrm{dl})$ were considered as diabetic and then two months late, those diabetic rats were used for the following two studies.

The first study as a pilot experiment was performed with two purposes: firstly to define whether diabetes impairs wound healing, and secondly to define whether aFGF can have similar or even better therapeutic effect on diabetes-impaired wound healing than recombinant human basic fibroblast growth factor (bFGF) as we found in clinical setting. ${ }^{19)}$ For this end, diabetic and age-matched nondiabetic (control) rats were anesthetized with $5 \%$ Chloral Hydrate $(0.5 \mathrm{ml} / \mathrm{kg}$ body weight $)$ and depilated using $8 \%$ sodium sulfide. A round marker (diameter $2.4 \mathrm{~cm}, 4.52 \mathrm{~cm}^{2}$ ) was made on the back of rats with methylrosanilinium chloride. The fullthickness skin in the marked area was snipped with a surgical scissors and the wound area was covered with sterile petrolatum gauze. The rats were divided into 4 groups: control (non-diabetic rats treated with saline, $n=6$ ), diabetes (diabetic rats treated with saline, $n=9$ ), diabetes/bFGF (diabetic rats treated with aFGF at $20 \mu \mathrm{g} / \mathrm{cm}^{2}, n=10$ ), and diabetes/bFGF (diabetic rats treated with bFGF at $20 \mu \mathrm{g} / \mathrm{cm}^{2}, n=10$ ). One single administration of various treatments was given every other day, respectively.

After defining the success of animal mode showing that diabetes impairs skin wound healing and both bFGF and aFGF are effective in improving diabetes-impaired wound healing in the above pilot study, the second study was performed to directly compare the therapeutic effects of FGF alone with the combination of it with $\mathrm{Zn}$ and/or G-CSF in only diabetic rats. We did not include non-diabetic group in this study since each of the approach used in the combination has been used extensively to non-diabetic animals. ${ }^{19,33,39-41)}$ In these previous studies, application of the exogenous growth factors or $\mathrm{Zn}$ did not always stimulate the wound healing process, for which one of the reasons is because normal tissue is not defect for these growth factors or nutrients such as $\mathrm{Zn} .{ }^{42}$ Diabetic induction by STZ and the full-thickness skin wound for the diabetic rats was made as described in the pilot study. Therefore, 114 diabetic rats at 2 months after the onset of diabetes were randomly divided into 8 groups, 14 rats per group, and aFGF $\left(20 \mu \mathrm{g} / \mathrm{cm}^{2}\right)$, bFGF $\left(20 \mu \mathrm{g} / \mathrm{cm}^{2}\right)$, insulin $(1 \mathrm{U} / 300 \mathrm{~g})$, G-CSF $\left(1800 \mathrm{U} / \mathrm{cm}^{2}\right), 0.01 \% \mathrm{ZnSO}_{4}\left(0.1 \mathrm{ml} / \mathrm{cm}^{2}\right)$, vehicle $\left(0.1 \mathrm{ml} / \mathrm{cm}^{2}\right)$, aFGF/G-CSF/ZnSO ${ }_{4}$ and $\mathrm{aFGF} / \mathrm{G}-$ CSF were administrated every other days, respectively.

\section{Diabetic Ulcer Healing Status Monitoring -} The wound size was monitored using a digital camera at day $3,6,9,12,15,18,21$ and 28 after injury and drugs administration and quantified using image-pro plus 5.2 software according to proportional reference scale. Meanwhile, the wound healing time including average time to reach $100 \%$ healing in each group was also calculated.

Histopathological Examination- At days 7, 14, 21 after treatment, rats were sacrificed to collect 2 pieces of dorsal skin from wound center and wound edge areas to perform histopathological examination according to our previous report. ${ }^{38)}$ There was no histological observation at 28 days since at this time-point, most of the treatment groups have $100 \%$ healed wound. Histopathological analysis focused on the changes of thickness of epithelia, granulation tissue formation and neovascularization.

Capillary Density — Micro vessels with diameter between $10 \mu \mathrm{m}$ to $50 \mu \mathrm{m}$ were counted in high power field (400 fold) randomly, 9 fields per section. The capillary density was expressed as micro vessel number per high power field (400 fold). 
Collagen Staining — Collagen in intercellular substance and cell matrix were stained by Masson staining method. Sections from wound center area were dewaxed and rehydrated conventionally, placed in Masson solution (acid fuchsin $1 \mathrm{~g}$, ponceau red $1 \mathrm{~g}$ and orange $\mathrm{G} 2 \mathrm{~g}$ in $100 \mathrm{ml} 0.5 \%$ acetic acid) for $5 \mathrm{~min}$ and then in $0.5 \%$ acetic acid for $1 \mathrm{~min}$, and rinsed in deionized water before placing in phosphomolybdic acid (2\%) for $5 \mathrm{~min}$. Subsequently, sections were immersed in $0.5 \%$ bright green for $5 \mathrm{~min}$, and rinsed in $0.5 \%$ acetic acid, $95 \%$ ethanol, and absolute ethanol in turn, immersed in xylene for $10 \mathrm{~min}$, and mounted with resin. Collagen fibers were stained blue, cytoplasm and erythrocyte were stained red, and nuclei were stained bluish brown.

Immunohistochemical Staining — Tissue paraffin sections $(5 \mu \mathrm{m})$ from edge area were used for proliferating cell nuclear antigen (PCNA, 1:50), tissue inhibitor of metalloproteinase 1 (TIMP-1, $1: 100$ ), and matrix metalloproteinase 1 (MMP-1, $1: 100)$ immunohistochemical staining according to the methods as our previous report. ${ }^{38)}$ The expression of PCNA, TIMP-1 and MMP-1 was quantified using image-pro plus 5.2 software. Three high-power fields (400 fold) per slide were counted for PCNA, TIMP-1 and MMP-1 positive cells, for which three slides from each of eight to fourteen animals per treatment group were analyzed. The expression of PCNA, TIMP-1 and MMP-1 was expressed as percentage of positive cells in the total cells under 400 fold high-power fields.

Statistical Analysis — All data displayed as mean \pm S.D., analyzed by one-way analysis of vari- ance (ANOVA) and Chi-square test. LSD- $t$ test was used to compare differences between groups, and the rate difference between groups was compared using partitions of $\chi^{2}$ method. SPSS12.0 software was used for the rest statistical tests. Differences were considered to be significant at $p<0.05$.

\section{RESULTS}

\section{Preparation of Diabetic Rats}

Rats receiving STZ resulted in significant elevation in blood glucose level ( $\geq 16.7 \mathrm{mmol} / \mathrm{l})$ after $1 \mathrm{wk}$ (Fig. 1A), which was sustained throughout the duration of the study. On average, rats had a mean body weight of $200.8 \pm 7.3 \mathrm{~g}$ at the beginning of the study, the body weight of diabetic rat increased slowly, and reached to about one half of the body weight of non-diabetic rats at 2 months after diabetes onset (Fig. 1B), which is consistent with our previous studies.

\section{Diabetes-delayed Skin Wound Healing and its Improvement by FGF}

To ensure that diabetes suppresses skin wound healing and this diabetic suppression of skin wound healing can be improved by FGF, a pilot study was performed. Skin wound was made for both diabetic rats at 2 months after the onset of diabetes and age-matched non-diabetic rats, and diabetic rats were randomly divided into four groups as shown in Fig. 2. In non-diabetic rats treated with saline (Control), the skin wound was completely healed around 15 days after injury (Fig. 2A, B) while it was signif-
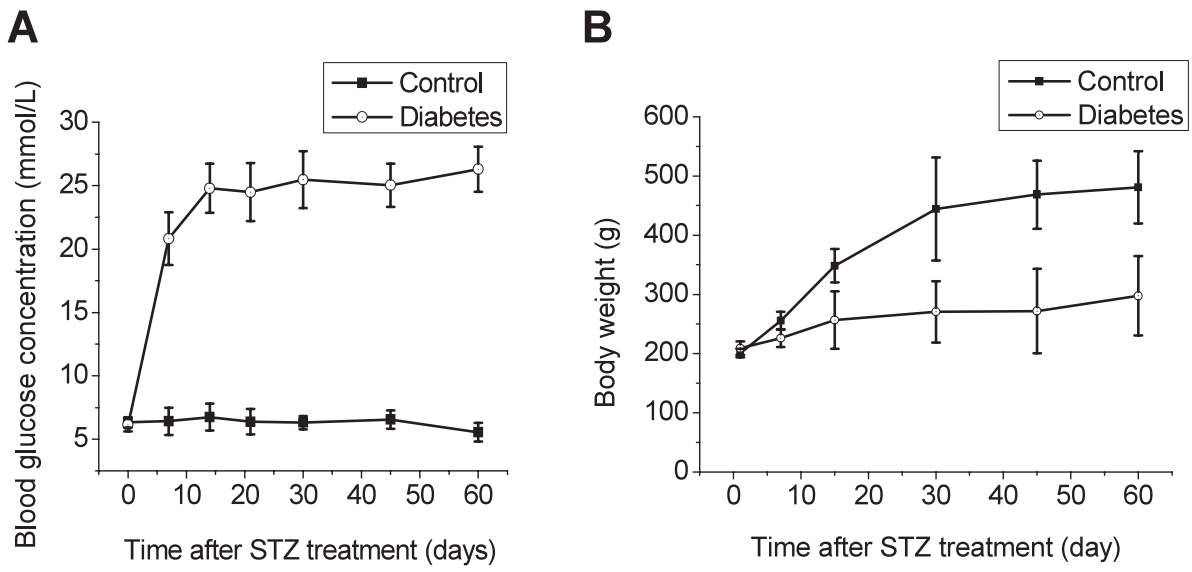

Fig. 1. Changes in Blood Glucose and Body Weight in Diabetic Rats

Blood glucose was elevated and maintained at high level (A), and the body weight had no significant gain at indicated time points in two months after STZ induction compared to control (B). 

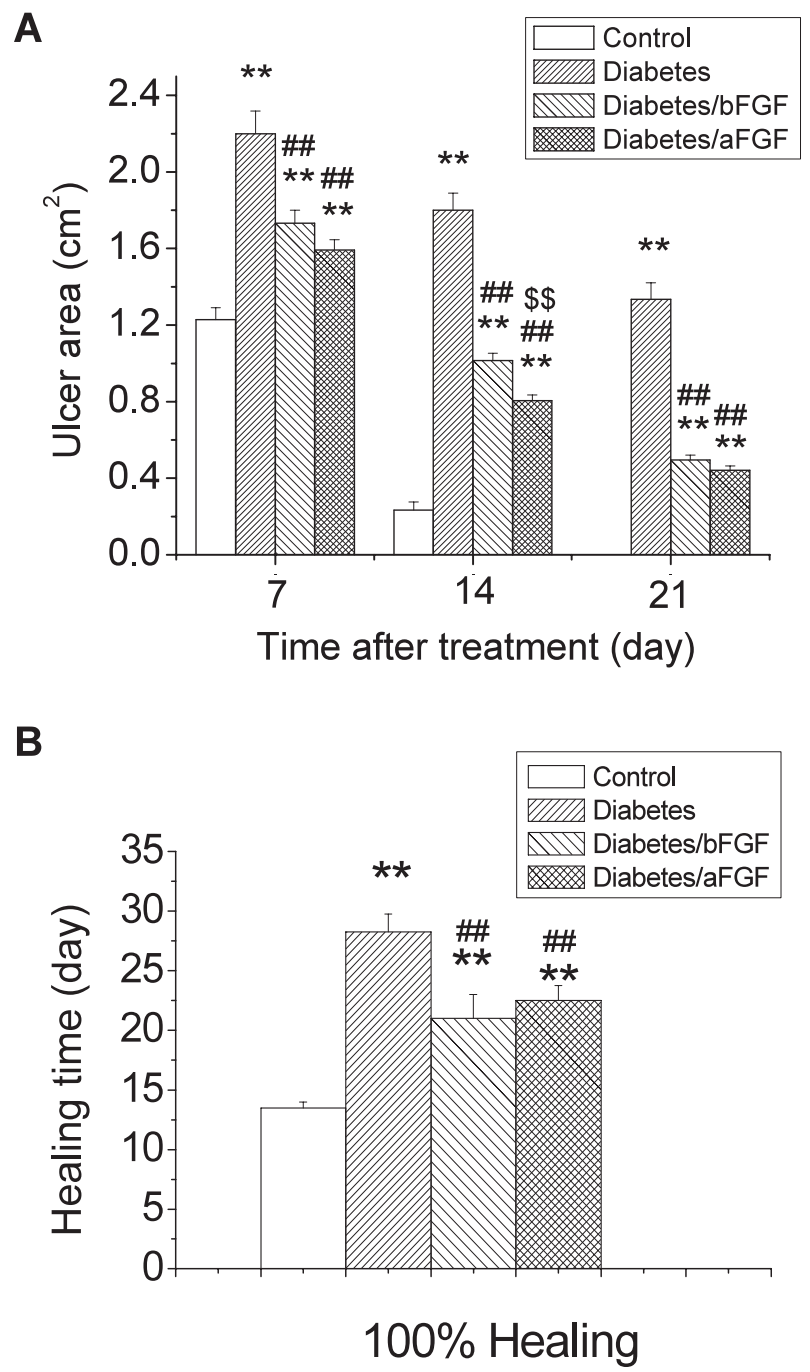

Fig. 2. Diabetic Ulcer Wound Model

Full-thickness excisional wounds (diameter $1.8 \mathrm{~cm}, 2.54 \mathrm{~cm}^{2}$ ) were created on the back of male diabetic rats. The wound closure process in normal and diabetic rats with different treatments was monitored and the residual ulcer area after drug administration was demonstrated (A), and the $100 \%$ healing time was calculated. ${ }^{* *}: p<0.01$, vs. Control; \#\#: $p<0.01, v s$. Diabetes; ${ }^{\$}: p<0.01, v s$. bFGF.

icantly delayed in saline-treated diabetic rats (Diabetes) until 28 days after injury (Fig. 2A, B). Results also showed that treatment with bFGF (Diabetes/bFGF) as the commonly-used approach for diabetic ulcer in China significantly accelerated the wound healing, shown by reducing ulcer sizes at indicted time points (Fig. 2A) and completely healing time (Fig. 2B) as compared to Control. Furthermore, it should be noted that aFGF was also effective in accelerating diabetic wound healing, which is similar to or even better than bFGF (Fig. 2).

\section{aFGF/G-CSF/Zn Enhanced Diabetic Ulcer Clo- sure}

In the second study, we included bFGF as the positive control group of commonly-used treatment protocol and compared aFGF alone with its combination with other approaches because the therapeutic effect on diabetic wound healing of aFGF was similar to or even better than bFGF (Fig. 2). ${ }^{19}$ ) Results showed that on the indicated time points, aFGF/G-CSF/ZnSO ${ }_{4}$ had the best wound healing effect on diabetic ulcer $(p<0.01)$, and aFGF/GCSF showed good therapeutic effect although less than that of aFGF/G-CSF/ZnSO $4(p<0.05)$. Treatment with aFGF showed similar therapeutic effect to that of positive control bFGF ( $p>0.05$ ), but all single drug treatment groups had less therapeutic effect on ulcer healing than combined treatment (Fig. 3A).

We also analyzed ulcer healing time and found that only aFGF/G-CSF/ZnSO ${ }_{4}$ group had significant shorter healing time ( $20 \pm 1.15$ days for $100 \%$ healing) than that of group treated with bFGF, insulin, $\mathrm{ZnSO}_{4}$ or vehicle alone treatment $(p<0.05)$, respectively (Fig. 3B).

\section{aFGF/G-CSF/ZnSO 4 Improved Skin Regenera- tion and Remodeling}

We next examined the histopathology of ulcer skin in diabetes. The representative microscopic findings were shown in Fig. 4A. Compared with single treatment groups and vehicle group, aFGF/G$\mathrm{CSF} / \mathrm{ZnSO}_{4}$ treatment displayed hyperplasia of epidermis, improved fibroblast cell proliferation, accompanied with massive capillary vascular bud formation, and increased density of vascular bud in ulcer central area. There was significantly regenerating and proliferating area along with rare muscle fibers were found in the epidermis (Fig. 4A).

The pathological examination revealed that in wound margin of aFGF/G-CSF/ZnSO 4 treated rats, the corneous layer and skin appendages such as hair follicles, medulla of hair and sebaceous gland were more evident than other groups on day 14. Granular layer hypotrophy disappeared, and the basal layer became rarefaction. Epidermis was filled with collagen, and the diameter of microvessels became bigger. However, there were no significant differences between aFGF/G-CSF/ZnSO 4 and aFGF/GCSF treatment groups (Fig. 4A).

We further counted the microvessel density, which showed that $\mathrm{aFGF} / \mathrm{G}-\mathrm{CSF} / \mathrm{ZnSO}_{4}$ induced angiogenesis and revascularization signifi- 

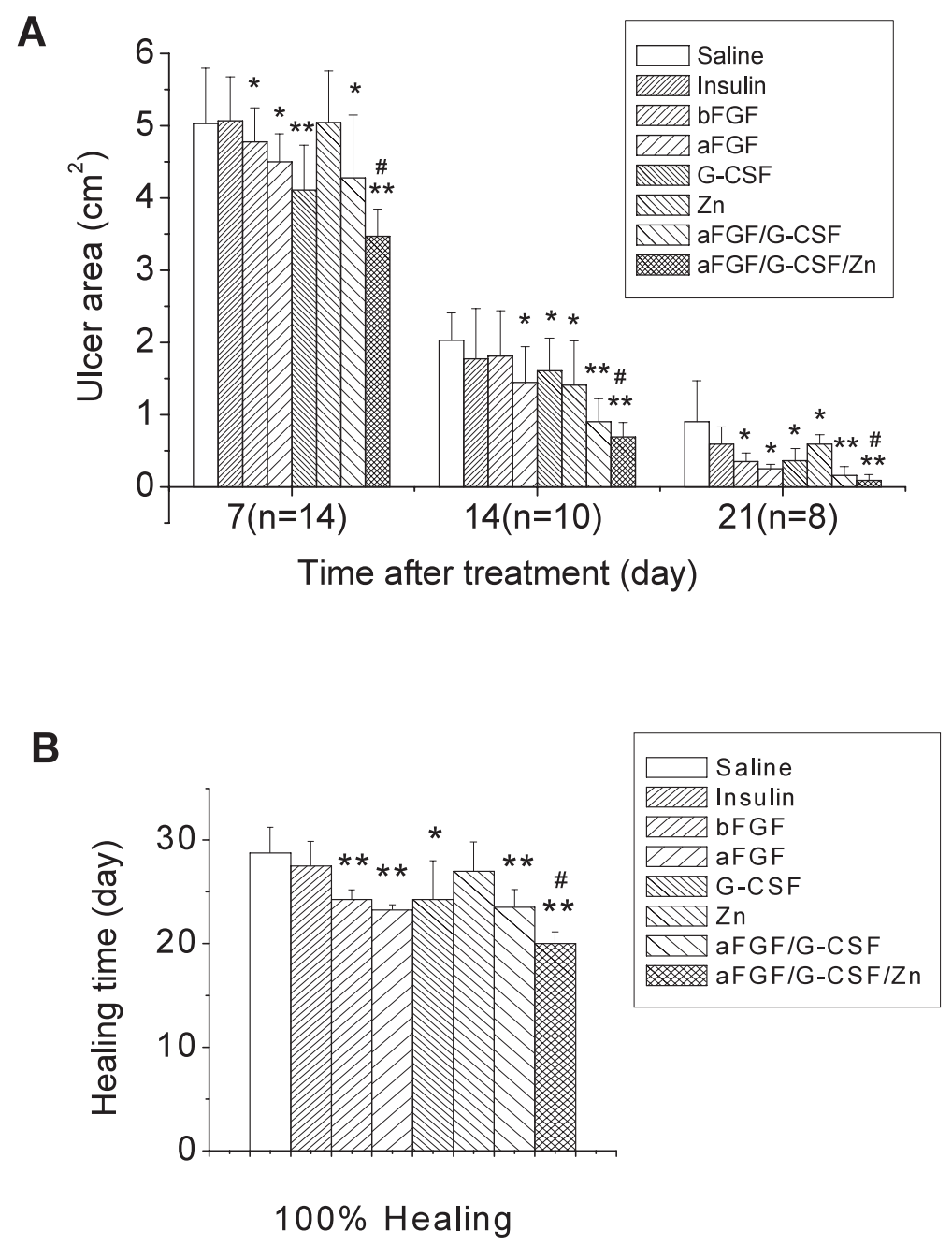

Fig. 3. Diabetic Ulcer Wound Closure

Full-thickness excisional wounds about $2.4 \mathrm{~cm}$ in diameter were created on the back of male diabetic rats. The wound closure process was monitored and the residual ulcer area after drug administration was demonstrated (A), and the $100 \%$ healing time was calculated. ${ }^{*}$ or ${ }^{* *}: p<0.05$ or 0.01 , vs. Saline; : $p<0.05$, vs. aFGF, G-CSF, Zn, or aFGF/G-CSF.

cantly. The capillary density were $19.50 \pm 3.11$, $16.67 \pm 1.15,29.00 \pm 3.61$ on day 7,14 and 21 respectively, which were significantly higher than that of positive control [bFGF: $5.5 \pm 0.58,11.33 \pm 0.57$ and $14.67 \pm 1.15$ on day 7,14 and $21(p<0.01)$ ]. Compared with bFGF, G-CSF and aFGF/G-CSF also induced higher quantity of microvessels $(p<$ 0.05). However, no statistical differences were found among aFGF/G-CSF/ZnSO 4 , aFGF/G-CSF and G-CSF groups. From all of the drug treated groups we found that the microvessel gradual formation accompanied by ulcer healing progression (Fig. 5).

Collagen was stained using Masson method, and collagen fiber was stained brilliant green and other issues were purple red or golden yellow. It was found that $\mathrm{aFGF} / \mathrm{G}-\mathrm{CSF} / \mathrm{ZnSO}_{4}$ induced fast epidermal cell proliferation and differentiation, although there were no obvious differences between experimental groups on 7 day after treatment, but on day 14 and 21 day, aFGF/G-CSF/ZnSO 4 group had fewer nuclei of fibroblast and more collagen fiber than other groups (Fig. 6).

\section{aFGF/G-CSF/Zn Enhanced Epidermal Cell Pro- liferation}

The proliferation of skin cells was detected by PCNA staining. We found that both aFGF/G$\mathrm{CSF} / \mathrm{ZnSO}_{4}$ and $\mathrm{aFGF} / \mathrm{G}-\mathrm{CSF}$ induced significant skin cell proliferation on day 7,14 and 21 compared to vehicle control $(p<0.05)$. G-CSF group had lower PCNA positive rate than positive control bFGF $(p<0.05)$. All other drug treated groups had significant higher PCNA positive rates than ve- 


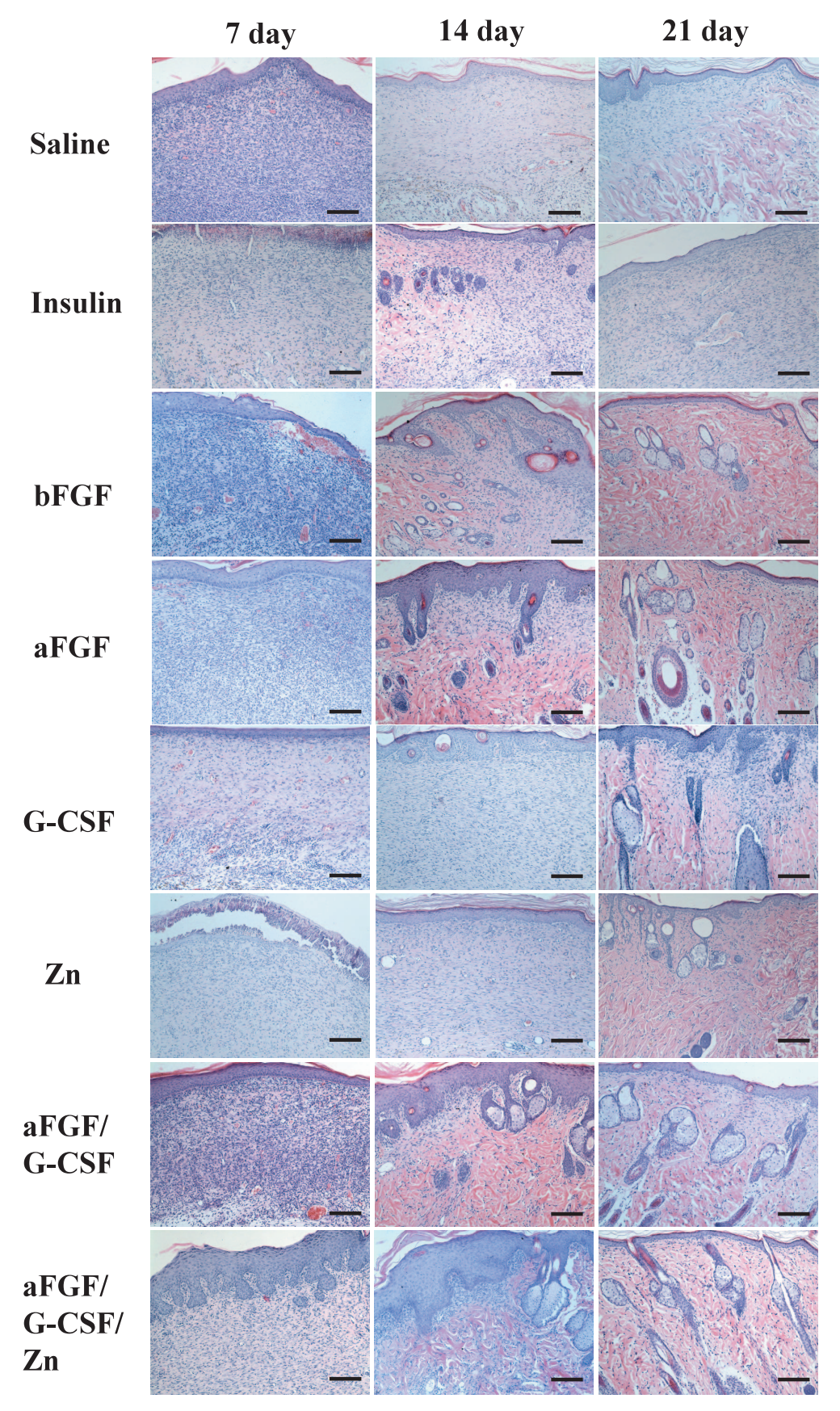

Fig. 4. Diabetic Ulcer Wound Healing

$\mathrm{H} \& \mathrm{E}$ staining indicated that aFGF, G-CSF and $\mathrm{ZnSO}_{4}$ combined administration induced obvious fibroblast and epidermis proliferation, and capillary bud formation. Stratum granulosum became hypertrophia and loose, stratum basa became compact in ulcer central zone and rare muscle fiber observed at corium. In wound edge area, stratum corneum and skin appendages regenerated earlier, sebaceous gland cells became large, hair follicles and medulla of hair stained deeply, and plenty of collagen fibers filled in corium $(\mathrm{Bar}=50 \mu \mathrm{m})$.

hicle control group $(p<0.05)$. At the same time, we found there was an obvious time-dependant PCNA expression pattern in all of the drug treatment groups, which was relative high on day 7 , highest on day 14 and lower on day 21 (Fig. 7A). These findings were consistent with ulcer healing progression.
aFGF/G-CSF/ZnSO 4 Down-regulated the Expression of MMP-1 and Up-regulated the Expression TIMP-1

The expression of TIMP-1 and MMP-1 were assayed by immunohistochemical staining. No statistical difference was found in MMP-1 expressions among different treatment groups on day $7(p>$ 


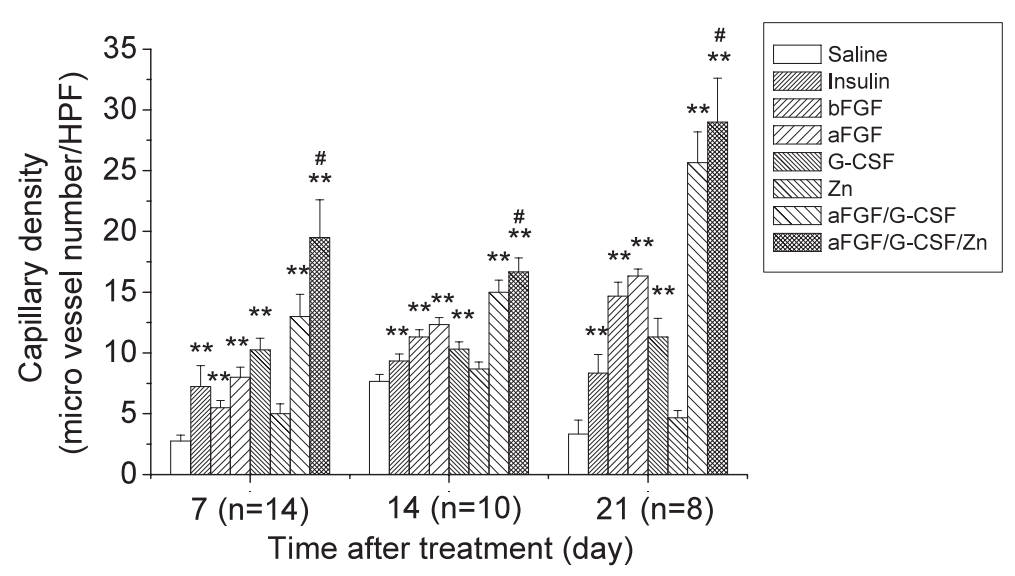

Fig. 5. Diabetic Ulcer Microvessel Regeneration

The quantity of blood capillary (diameter: $10-50 \mu \mathrm{m}$ ) was counted on H\&E stained sections on day 7, 14 and 21 after drug administration (B). ${ }^{* *}$ : $p<0.01$, vs. Saline; ${ }^{\#}: p<0.05$, vs. aFGF, G-CSF, Zn, or aFGF/G-CSF.

0.05). aFGF/G-CSF/ZnSO 4 group had the lowest MMP-1 expression at all indicated time points, and the relative expression level was about one third of positive control (bFGF) and aFGF/G-CSF group on day 14 and 21. MMP-1 expression in G-CSF group was comparable to that of positive control, and lower than that of vehicle control, but there were no obvious statistical differences among them $(p>0.05)$. From the above results, we found that the expression of MMP-1 had a time-dependant changing pattern along with ulcer healing processes, the expression maintained at lower level at incipient stage ( 7 day), then increased to peak (day 14), and gradually decreased to a relative lower level at advanced stage (day 21) (Fig. 7B).

TIMP-1 is an important factor to regulate the function of MMPs. The results demonstrated that aFGF/G-CSF/ZnSO ${ }_{4}$ induced a highest expression level of TIMP-1 among different treatment groups in the present study, and the relative levels were $15.00 \pm 1.53,34.33 \pm 9.29,46.67 \pm 7.09$ on day 7 , 14 and 21 respectively. The expression level of TIMP-1 in aFGF/G-CSF group was about half of $\mathrm{aFGF} / \mathrm{G}-\mathrm{CSF} / \mathrm{ZnSO}_{4}$ treatment group, and had no significant differences from positive and vehicle control group on day 14 ( $p>0.05)$. Zn had obviously influence on TIMP-1 expression $(p<0.05)$. However, G-CSF had no significant effect on the expression of TIMP-1 on day 7, 14 and 21 as compared with positive control bFGF and vehicle control group $(p>0.05)$ (Fig. 7C).

Because TIMP-1 is a negative regulator of MMP-1, we further calculated the ratios of MMP1/TIMP-1 (Fig. 7D). We found that the ratios of combination treatment groups were significant lower than that of single treatment groups $(p<$ 0.05 ), and the ratios of single treatment groups were significant lower than that of vehicle control group $(p<0.05)$.

\section{DISCUSSION}

Diabetic skin ulcer is one of the severe diabetic complications frequently found in diabetic patients. The mechanisms of this diabetic complication are complicated and remain unclearly understood. How to efficiently treat this complication is still a challenge to clinics. ${ }^{43)}$

Emerging evidence indicates that the mechanisms responsible for diabetic foot or skin ulcers may include at least four aspects, blood capillary pathological changes, impaired fibroblast proliferation, extracellular matrix formation and remolding disorders, and peripheral nerve abnormality. ${ }^{44-46)}$ For these four pathogenic changes, hyperglycemia seems the major cause. ${ }^{7)}$ However, this is not supported by our present study since only control blood glucose level in diabetic rats by injecting longacting insulin could not promote the diabetic ulcer healing processes compared with vehicle control group.

Normally, wounds healing process can be divided into several stages with different characteristics: at incipient stage after injury, a few kinds of inflammation factors moved toward wound area and formed inflammatory infiltration to eliminate injury factor and infection; ${ }^{47)}$ in the middle stage, 


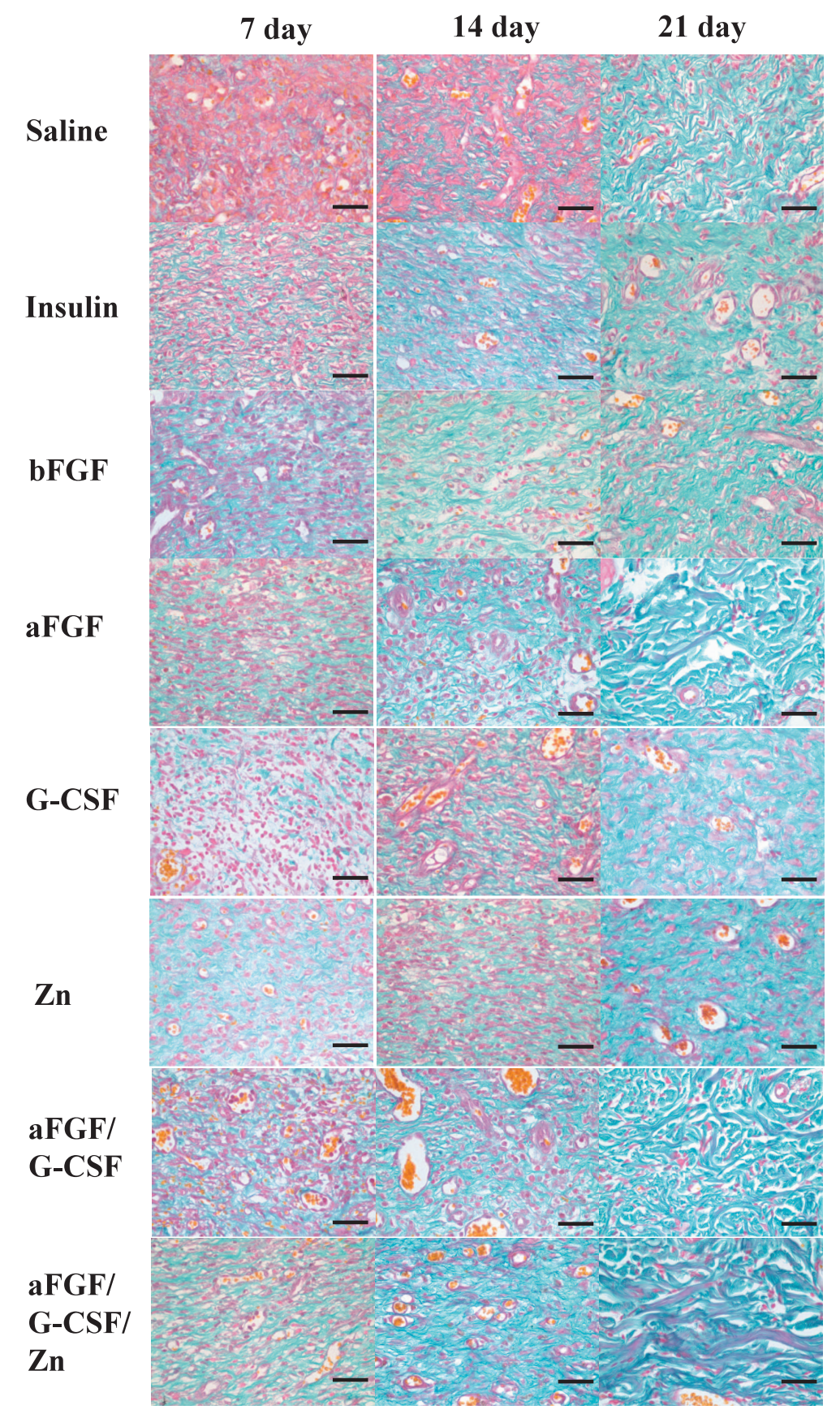

Fig. 6. The Collagen Fiber in Wound Area was Stained Using Masson Trichrome Staining on Day 7, 14 and 21 after Treatments The staining results showed that wounds developed vascular granulation tissue, tended to be populated with inflammatory cells and fibroblasts, and partially formed newly formed epithelium at the edge of the wounds in all of the 8 groups on day 7 after wounding, while on day 14, there were thicker and more highly organized collagen fibers deposition in wounds treated with $\mathrm{aFGF} / \mathrm{G}-\mathrm{CSF} / \mathrm{ZnSO}_{4}$, especially in $14(\mathrm{Bar}=50 \mu \mathrm{m})$.

blood capillary buds appeared and fibroblasts began to proliferate, and the blood capillary which was regarded as nutrition supplier provided material needed for wound healing ${ }^{48)}$ and fibroblast proliferation was required to repair the skin structural defect; in the final stage, tissue extracellular matrix formation and reconstruction was initiated. ${ }^{49)}$

Previous study showed that aFGF mainly en- hanced fibroblast mitosis to accelerate diabetic wound healing. ${ }^{38)}$ Furthermore, since the wound environments are acidic condition in the most time, especially with infection, the basic FGF would not be stable in this environment. It has been found to be better than bFGF in wound healing and myocardial preservation. ${ }^{39,50,51)}$ We also observed that aFGF provided a slightly better healing rate and ef- 
A

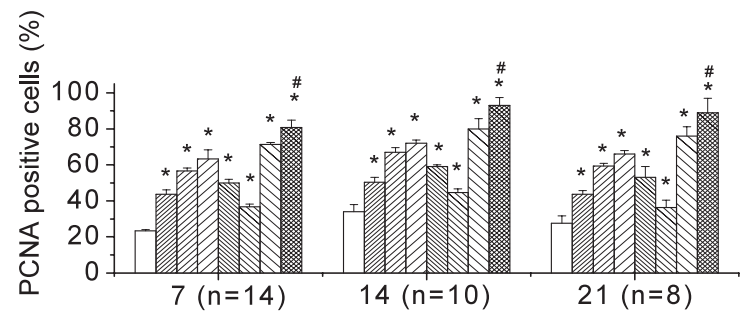

B

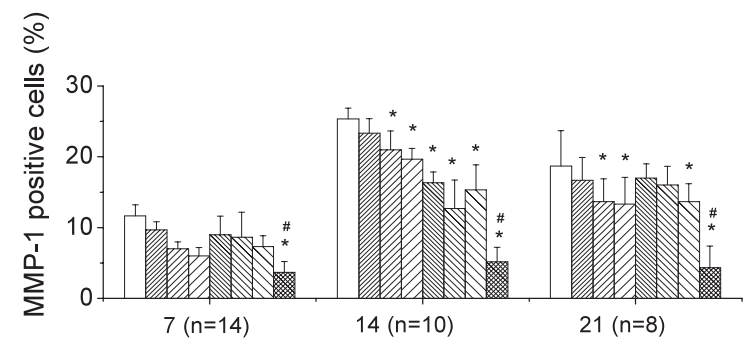

C

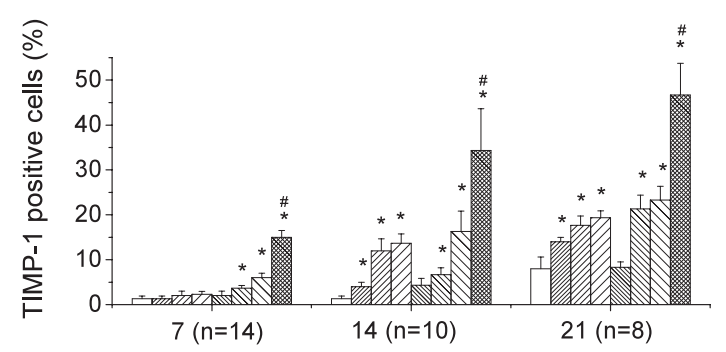

D

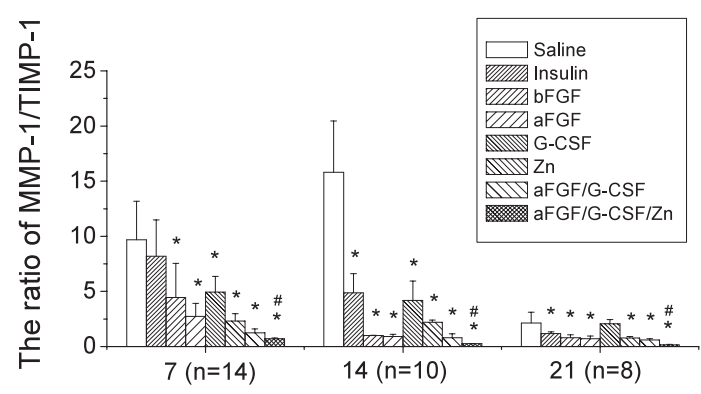

Fig. 7. Expression of PCNA (A), MMP-1 (B) and TIMP-1 (C) in Wound Tissue on both Day 7, 14 and 21 after Wounding in each Group were Evaluated by Immunohistochemistry Respectively, and the Ratios of MMP1/TIMP-1 were Also Calculated (D)

Definition of positive cells (\%) was given in the MATERIALS AND METHODS. ${ }^{*}: p<0.05$, vs. Saline; \# $: p<0.05$, vs. aFGF, G-CSF, Zn, or aFGF/G-CSF.

ficiency than bFGF in improving wound healing for diabetic patients. ${ }^{19)}$ In this experiment, aFGF group compared with positive control group had similar or even better curative effect, which is consistent with our previous study. ${ }^{19)}$ FGF can mainly enhance fibroblast mitosis or proliferation, but G-CSF is another important factor influence ulcer healing.

It has been shown that G-CSF can mobilize BMHPCs, thus promoting blood capillary regeneration and mobilizing neutrophil series. ${ }^{32)}$ Capillary vessel quantity assay displayed that G-CSF group override positive control group (bFGF group) and equivalent to $\mathrm{aFGF} / \mathrm{G}-\mathrm{CSF} / \mathrm{ZnSO}_{4}$ and $\mathrm{aFGF} / \mathrm{G}-\mathrm{CSF}$ groups in quantity. Our results support the previous finding that G-CSF is another important factor during ulcer healing period. ${ }^{33,52)}$

In the study with immunohistochemical stain results of MMPs and TIMP-1, we found the group combined using with $\mathrm{ZnSO}_{4}$ had lower MMPs and higher TIMP-1 expression. Zn links with Znbinding protein metallothionein as described in previous study. ${ }^{53,54)}$ Metallothionein exists in extracellular matrix and involves in regulation of MMPs and TIMPs which depend on $\mathrm{Zn}$ to influent collagen fiber decompounded or reconstruction. ${ }^{55)}$ Diabetes can disorder $\mathrm{Zn}$ homeostasis in body, which thus affects cell growth and proliferation, and increases oxidative stress. ${ }^{56,57)}$ Present experiment suggested that aFGF/G-CSF/ZnSO 4 group had better extracellular matrix formation, and consequently had better healing velocity and quality. It should be noted that the result in $\mathrm{ZnSO}_{4}$ group was compared with positive control group, indicating that $\mathrm{Zn}$ alone does not significantly stimulate wound healing, but has significantly synergic effect on the wound healing when it is combined with other factors.

In summary, the present study demonstrated that aFGF/G-CSF/ZnSO ${ }_{4}$ combination significantly improved diabetic ulcer healing, and the curative promotion was most likely associated with the functions of $\mathrm{ZnSO}_{4}$, aFGF, and G-CSF, i.e.: improving wound nutrient environments, fibroblast proliferation and stem cell-related blood capillary regeneration and extracellular matrix reformation and reconstruction. Therefore, this study opens a new road for us to explore the synergic combination of various effective approaches for an optimal therapy.

Acknowledgement The study was supported, in part, by grants from National Natural Scientific Foundation of China (30672495; 30870755, to Dr. X. K. Li), The Program of New Century Excellent Talents in University, Zhejiang Provincial Program for the Cultivation of High-Level Innovative Health Talents and Zhejiang Province Extremely Key Subject Building Funding "Pharmacology and Biochemical Pharmaceutics 2008.” (to Dr. X. K. Li), Zhejiang Provincial Medicine and Health Foundation (2006A099, to YT), and Juvenile Diabetes Research Foundation, International (5-2006-382, to Drs. L. Cai and X. K. Li), and also by a Start-Up fund for the Chinese-American Research Institute 
for Diabetic Complications from Wenzhou Medical College (to Drs. X. K. Li and L. Cai).

\section{REFERENCES}

1) Brem, H. and Tomic-Canic, M. (2007) Cellular and molecular basis of wound healing in diabetes. $J$. Clin. Invest., 117, 1219-1222.

2) Pavlovic, M. D., Milenkovic, T., Dinic, M., Misovic, M., Dakovic, D., Todorovic, S., Dakovic, Z., Zecevi, R. D. and Doder, R. (2007) The prevalence of cutaneous manifestations in young patients with type 1 diabetes. Diabetes Care, 30, 1964-1967.

3) Blakytny, R. and Jude, E. (2006) The molecular biology of chronic wounds and delayed healing in diabetes. Diabet. Med., 23, 594-608.

4) Blakytny, R., Jude, E. B., Martin Gibson, J., Boulton, A. J. and Ferguson, M. W. (2000) Lack of insulin-like growth factor 1 (IGF1) in the basal keratinocyte layer of diabetic skin and diabetic foot ulcers. J. Pathol., 190, 589-594.

5) Jude, E. B., Blakytny, R., Bulmer, J., Boulton, A. J. and Ferguson, M. W. (2002) Transforming growth factor-beta 1, 2, 3 and receptor type I and II in diabetic foot ulcers. Diabet. Med., 19, 440-447.

6) Brownlee, M. (2001) Biochemistry and molecular cell biology of diabetic complications. Nature, $\mathbf{4 1 4}$, 813-820.

7) Sowers, J. R. and Stump, C. S. (2004) Insights into the biology of diabetic vascular disease: what's new? Am. J. Hypertens, 17, 2S-6S.

8) Inoue, M., Ono, I., Tateshita, T., Kuroyanagi, Y. and Shioya, N. (1998) Effect of a collagen matrix containing epidermal growth factor on wound contraction. Wound Repair Regen., 6, 213-222.

9) Lee, A. R. (2005) Enhancing dermal matrix regeneration and biomechanical properties of 2nd degreeburn wounds by EGF-impregnated collagen sponge dressing. Arch. Pharm. Res., 28, 1311-1316.

10) Cheng, B., Liu, H. W., Fu, X. B., Sun, T. Z. and Sheng, Z. Y. (2007) Recombinant human plateletderived growth factor enhanced dermal wound healing by a pathway involving ERK and c-fos in diabetic rats. J. Dermatol. Sci., 45, 193-201.

11) Eldor, R., Raz, I., Ben Yehuda, A. and Boulton, A. J. (2004) New and experimental approaches to treatment of diabetic foot ulcers: a comprehensive review of emerging treatment strategies. Diabet. Med., 21, 1161-1173.

12) Duraisamy, Y., Slevin, M., Smith, N., Bailey, J., Zweit, J., Smith, C., Ahmed, N. and Gaffney, J. (2001) Effect of glycation on basic fibroblast growth factor induced angiogenesis and activation of associated signal transduction pathways in vascular endothelial cells: possible relevance to wound healing in diabetes. Angiogenesis, 4, 277-288.

13) Kloth, L. C. and Mcculloch, J. M. (2002) Wound Healing Alternatives in Management, FA Davis Company, Philadephia.

14) Muangman, P., Muffley, L. A., Anthony, J. P., Spenny, M. L., Underwood, R. A., Olerud, J. E. and Gibran, N. S. (2004) Nerve growth factor accelerates wound healing in diabetic mice. Wound Repair Regen., 12, 44-52.

15) Galiano, R. D., Tepper, O. M., Pelo, C. R., Bhatt, K. A., Callaghan, M., Bastidas, N., Bunting, S., Steinmetz, H. G. and Gurtner, G. C. (2004) Topical vascular endothelial growth factor accelerates diabetic wound healing through increased angiogenesis and by mobilizing and recruiting bone marrowderived cells. Am. J. Pathol., 164, 1935-1947.

16) Matuszewska, B., Keogan, M., Fisher, D. M., Soper, K. A., Hoe, C. M., Huber, A. C. and Bondi, J. V. (1994) Acidic fibroblast growth factor: evaluation of topical formulations in a diabetic mouse wound healing model. Pharm. Res., 11, 65-71.

17) Tsuboi, R. and Rifkin, D. B. (1990) Recombinant basic fibroblast growth factor stimulates wound healing in healing-impaired $\mathrm{db} / \mathrm{db}$ mice. J. Exp. Med., 172, 245-251.

18) Ito, K., Ito, S., Sekine, M. and Abe, M. (2005) Reconstruction of the soft tissue of a deep diabetic foot wound with artificial dermis and recombinant basic fibroblast growth factor. Plast. Reconstr. Surg., 115, 567-572.

19) Tan, Y., Xiao, J., Huang, Z., Xiao, Y., Lin, S., Jin, L., Feng, W., Cai, L. and Li, X. (2008) Comparison of the therapeutic effects between recombinant human acidic and basic fibroblast growth factors in wound healing in diabetic patients. J. Health Sci., 54, 423440.

20) Song, Y., Wang, J., Li, X. K. and Cai, L. (2005) Zinc and the diabetic heart. Biometals, 18, 325-332.

21) Mata, A. D., Marques, D., Rocha, S., Francisco, H., Santos, C., Mesquita, M. F. and Singh, J. (2004) Effects of diabetes mellitus on salivary secretion and its composition in the human. Mol. Cell. Biochem., 261, 137-142.

22) Bouda, M., Krizek, M., Malkusova, I., Motan, J., Pizinger, K. and Rusavy, Z. (1994) Zinc deficiency as a cause of dermatosis in a patient with type I diabetes and renal insufficiency. Vnitr. Lek., 40, 445447.

23) Agren, M. S., Soderberg, T. A., Reuterving, C. O., Hallmans, G. and Tengrup, I. (1991) Effect of topi- 
cal zinc oxide on bacterial growth and inflammation in full-thickness skin wounds in normal and diabetic rats. Eur. J. Surg., 157, 97-101.

24) Rostan, E. F., Debuys, H. V., Madey, D. L. and Pinnell, S. R. (2002) Evidence supporting zinc as an important antioxidant for skin. Int. J. Dermatol., 41, 606-611.

25) Lansdown, A. B. (2002) Metallothioneins: potential therapeutic aids for wound healing in the skin. Wound Repair Regen., 10, 130-132.

26) Loomans, C. J., De Koning, E. J., Staal, F. J., Rookmaaker, M. B., Verseyden, C., De Boer, H. C., Verhaar, M. C., Braam, B., Rabelink, T. J. and Van Zonneveld, A. J. (2004) Endothelial progenitor cell dysfunction: a novel concept in the pathogenesis of vascular complications of type 1 diabetes. Diabetes, 53, 195-199.

27) Kaji, T., Fujiwara, Y., Yamamoto, C., Sakamoto, M. and Kozuka, H. (1994) Stimulation by zinc of cultured vascular endothelial cell proliferation: possible involvement of endogenous basic fibroblast growth factor. Life Sci., 55, 1781-1787.

28) Fujiwara, Y. and Kaji, T. (1997) Zinc potentiates the stimulation by basic and acidic fibroblast growth factors on the proliferation of cultured vascular smooth muscle cells. Res. Commun. Mol. Pathol. Pharmacol., 97, 95-106.

29) Sivan-Loukianova, E., Awad, O. A., Stepanovic, V., Bickenbach, J. and Schatteman, G. C. (2003) CD34+ blood cells accelerate vascularization and healing of diabetic mouse skin wounds. J. Vasc. Res., 40, 368-377.

30) Fang, B., Shi, M., Liao, L., Yang, S., Liu, Y. and Zhao, R. C. (2003) Multiorgan engraftment and multilineage differentiation by human fetal bone marrow Flk1+/CD31-/CD34- Progenitors. J. Hematother. Stem Cell Res., 12, 603-613.

31) Canturk, N. Z., Vural, B., Esen, N., Canturk, Z., Oktay, G., Kirkali, G. and Solakoglu, S. (1999) Effects of granulocyte-macrophage colonystimulating factor on incisional wound healing in an experimental diabetic rat model. Endocr. Res., 25, 105-116.

32) Groves, R. W. and Schmidt-Lucke, J. A. (2000) Recombinant human GM-CSF in the treatment of poorly healing wounds. Adv. Skin Wound Care, 13, 107-112.

33) Mery, L., Girot, R. and Aractingi, S. (2004) Topical effectiveness of molgramostim (GM-CSF) in sickle cell leg ulcers. Dermatology, 208, 135-137.

34) Albertson, S., Hummel, R. P., 3rd., Breeden, M. and Greenhalgh, D. G. (1993) PDGF and FGF reverse the healing impairment in protein-malnourished dia- betic mice. Surgery, 114, 368-372; discussion 372363.

35) Greenhalgh, D. G., Sprugel, K. H., Murray, M. J. and Ross, R. (1990) PDGF and FGF stimulate wound healing in the genetically diabetic mouse. Am. J. Pathol., 136, 1235-1246.

36) Brown, R. L., Breeden, M. P. and Greenhalgh, D. G. (1994) PDGF and TGF-alpha act synergistically to improve wound healing in the genetically diabetic mouse. J. Surg. Res., 56, 562-570.

37) Kiritsy, C. P., Antoniades, H. N., Carlson, M. R., Beaulieu, M. T., D'andrea, M. and Lynch, S. E. (1995) Combination of platelet-derived growth factor-BB and insulin-like growth factor-I is more effective than platelet-derived growth factor-BB alone in stimulating complete healing of fullthickness wounds in "older" diabetic mice. Wound Repair Regen., 3, 340-350.

38) Wang, W., Lin, S., Xiao, Y., Huang, Y., Tan, Y., Cai, L. and Li, X. (2008) Acceleration of diabetic wound healing with chitosan-crosslinked collagen sponge containing recombinant human acidic fibroblast growth factor in healing-impaired STZ diabetic rats. Life Sci., 82, 190-204.

39) Fredj-Reygrobellet, D., Plouet, J., Delayre, T., Baudouin, C., Bourret, F. and Lapalus, P. (1987) Effects of aFGF and bFGF on wound healing in rabbit corneas. Curr. Eye Res., 6, 1205-1209.

40) Lansdown, A. B., Mirastschijski, U., Stubbs, N., Scanlon, E. and Agren, M. S. (2007) Zinc in wound healing: theoretical, experimental, and clinical aspects. Wound Repair Regen., 15, 2-16.

41) Zhang, X. J., Wu, X., Wolf, S. E., Hawkins, H. K., Chinkes, D. L. and Wolfe, R. R. (2007) Local insulin-zinc injection accelerates skin donor site wound healing. J. Surg. Res., 142, 90-96.

42) Norman, J. N., Rahmat, A. and Smith, G. (1975) Effect of supplements of zinc salts on the healing of granulating wounds in the rat and guinea pig. $J$. Nutr., 105, 815-821.

43) Cavanagh, P. R., Lipsky, B. A., Bradbury, A. W. and Botek, G. (2005) Treatment for diabetic foot ulcers. Lancet, 366, 1725-1735.

44) Cullen, B. (2002) The role of oxidized regenerated cellulose/collagen in chronic wound repair. Part 2. Ostomy Wound Manage., 48, 8-13.

45) Neely, A. N., Clendening, C. E., Gardner, J. and Greenhalgh, D. G. (2000) Gelatinase activities in wounds of healing-impaired mice versus wounds of non-healing-impaired mice. J. Burn. Care Rehabil., 21, 395-402.

46) Armstrong, D. G. and Jude, E. B. (2002) The role of matrix metalloproteinases in wound healing. J. Am. 
Podiatr. Med. Assoc., 92, 12-18.

47) Mi, Q., Riviere, B., Clermont, G., Steed, D. L. and Vodovotz, Y. (2007) Agent-based model of inflammation and wound healing: insights into diabetic foot ulcer pathology and the role of transforming growth factor-beta1. Wound Repair Regen., 15, 671682.

48) Krishnan, S. T., Baker, N. R., Carrington, A. L. and Rayman, G. (2004) Comparative roles of microvascular and nerve function in foot ulceration in type 2 diabetes. Diabetes Care, 27, 1343-1348.

49) Medina, A., Scott, P. G., Ghahary, A. and Tredget, E. E. (2005) Pathophysiology of chronic nonhealing wounds. J. Burn. Care Rehabil., 26, 306-319.

50) Li, X. K., Lin, Z. F., Li, Y., Hu, S., Tan, Y., Huang, Z., Meng, J., Liang, L. M., Xiao, J., Qu, J. and Cai, L. (2007) Cardiovascular protection of nonmitogenic human acidic fibroblast growth factor from oxidative damage in vitro and in vivo. Cardiovasc. Pathol., 16, 85-91.

51) Lopez, J. J., Edelman, E. R., Stamler, A., Hibberd, M. G., Prasad, P., Thomas, K. A., Disalvo, J., Caputo, R. P., Carrozza, J. P., Douglas, P. S., Sellke, F. W. and Simons, M. (1998) Angiogenic potential of perivascularly delivered aFGF in a porcine model of chronic myocardial ischemia. Am. J. Physiol., 274, H930-H936.
52) Hinchliffe, R. J., Valk, G. D., Apelqvist, J., Armstrong, D. G., Bakker, K., Game, F. L., Hartemann-Heurtier, A., Londahl, M., Price, P. E., Van Houtum, W. H. and Jeffcoate, W. J. (2008) A systematic review of the effectiveness of interventions to enhance the healing of chronic ulcers of the foot in diabetes. Diabetes Metab. Res. Rev., 24(Suppl 1), S119-S144.

53) Ovington, L. G. (2002) Overview of matrix metalloprotease modulation and growth factor protection in wound healing. Part 1. Ostomy Wound Manage., 48, 3-7.

54) Schwartz, J. R., Marsh, R. G. and Draelos, Z. D. (2005) Zinc and skin health: overview of physiology and pharmacology. Dermatol. Surg., 31, 837-847.

55) Nwomeh, B. C., Liang, H. X., Cohen, I. K. and Yager, D. R. (1999) MMP-8 is the predominant collagenase in healing wounds and nonhealing ulcers. J. Surg. Res., 81, 189-195.

56) Schlienger, J. L., Grunenberger, F., Maier, E. A., Simon, C., Chabrier, G. and Leroy, M. J. (1988) Disorders of plasma trace elements in diabetes. Relation to blood glucose equilibrium. Presse Med., 17, 1076-1079.

57) Mateo, M. C., Bustamante, J. B. and Cantalapiedra, M. A. (1978) Serum, zinc, copper and insulin in diabetes mellitus. Biomedicine, 29, 56-58. 